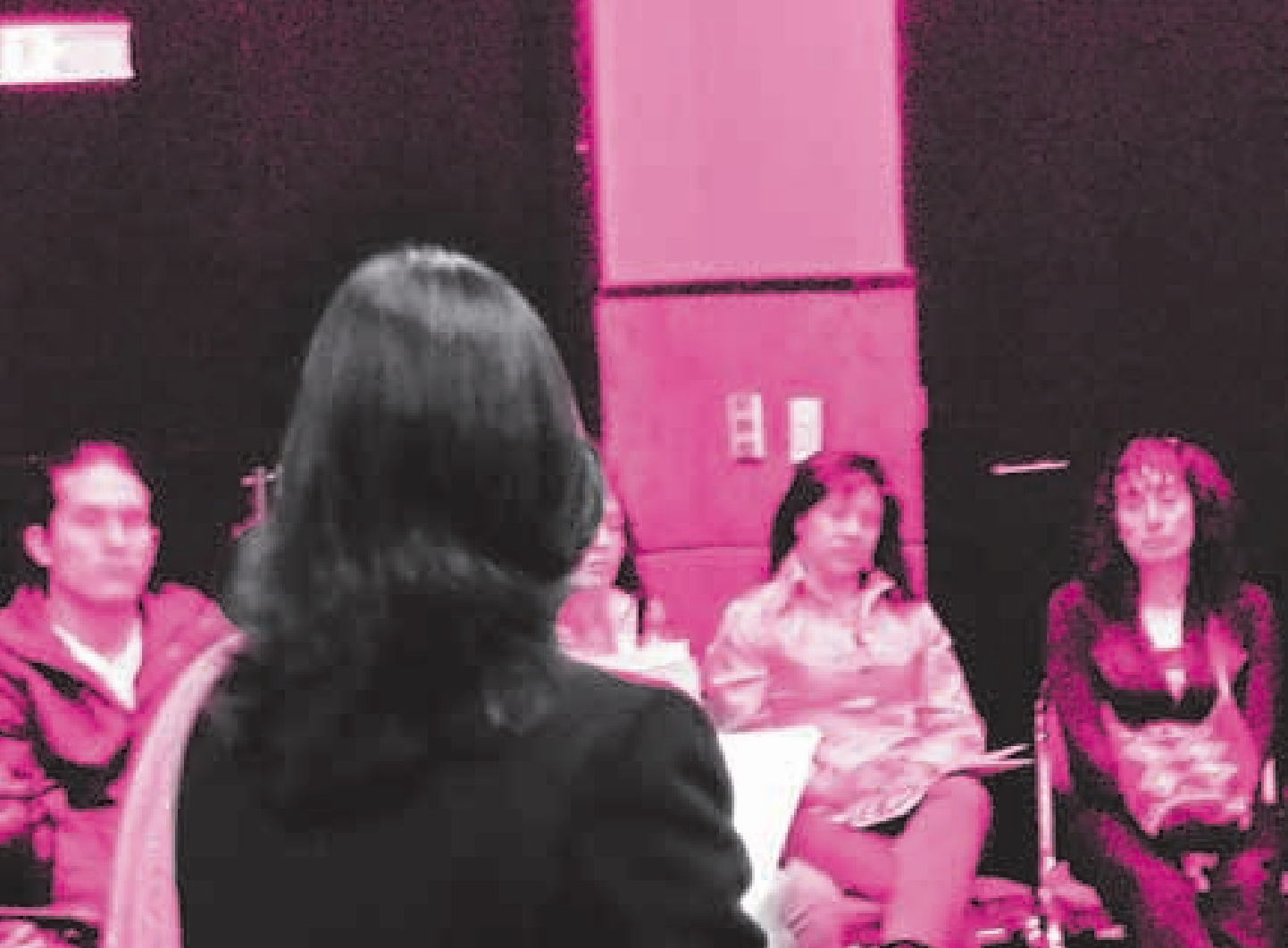

\title{
El desarrollo de conocimiento didáctico del contenido
}

En profesores de ciencias, noveles y expertos, a partir de la inmersión en equipos colaborativos de trabajo*

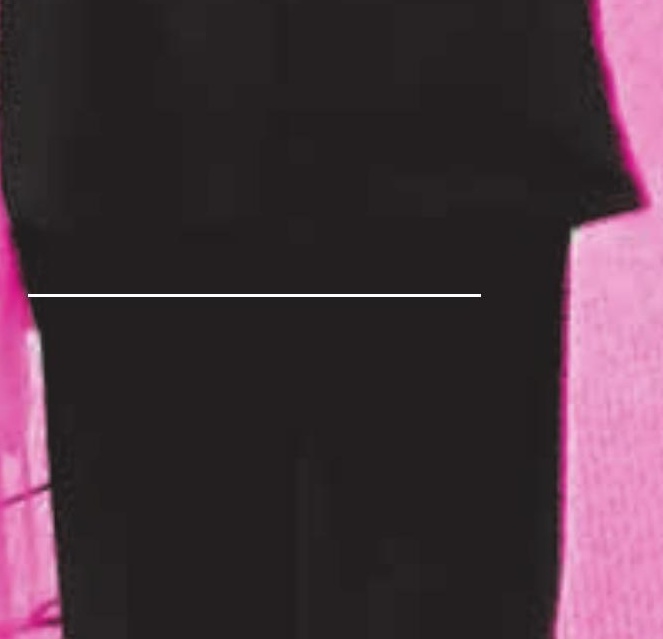

Carlos Javier Mosquera Suárez María Elvira Sánchez Hernández Carlos Andrés Solano Comezaquira * Este artículo hace parte de los resultados del Proyecto de Investigación "Estrategias para el favorecimiento de cambios didácticos en profesores de química, en formación inicial y en ejecicio a partir de la reflexión sobre la práctica", financiado por el Centro de Investigaciones y Desarrollo Científico, de la Universidad Distrital. 


\section{Resumen}

En esta investigación se pretende mostrar la experiencia de una estrategia innovadora en metodologías de enseñanzaaprendizaje de las ciencias, aplicada en el Programa de Formación de Profesores del Proyecto Curricular de Licenciatura en Química de la Universidad Distrital Francisco José de Caldas en Bogotá - Colombia. El propósito ha sido el de generar una nueva perspectiva de profesionalización de la actividad docente mejorando los sistemas de Formación Inicial y Continuada de Profesores de Ciencias. Para lograr esto, se identificó el Conocimiento Didáctico del Contenido de profesores expertos y novatos y se hizo seguimiento a los resultados de la implementación de la estrategia con el apoyo del Software ATLAS.ti ${ }^{\circledR}$ para determinar los cambios en estos conocimientos, para establecer vínculos entre los Profesores noveles y expertos con un deseable de referencia y para finalmente generar cambios y procesos de autorregulación de las prácticas docentes.

\section{Palabras claves}

Conocimiento didáctico del contenido, formación inicial, formación continuada, docente experto y novel, equipos colaborativos de trabajo.

\section{Abstract}

This research aims to show the experience of an innovative approach in teaching-learning of science, applied at the Teacher Training Program Curriculum Degree Project in Chemistry from the Universidad Distrital Francisco José de Caldas in Bogotá - Colombia. The propose was to generate a new perspective on professionalization of teaching by improving the systems of initial and ongoing training of science teachers. To achieve this, we identified the pedagogical content knowledge of experienced teachers and novices and tracked the results of the implementation of the strategy supported by ATLAS.ti ${ }^{\circledR}$ Software to determine changes in this knowledge, to establish links between new teachers and experts with a desirable reference and to ultimately bring about change and processes of self-teaching practices.

Key words

Pedagogical content knowledge, Initial Training and Continuing Education, novice and experienced teacher, Collaborative Working Team.

\section{Abstração}

Esta pesquisa visa mostrar a experiência de uma abordagem inovadora no ensino-aprendizagem das ciências, aplicados no Programa de Formação do Projeto Professor Licenciatura Curriculum em Química pela Universidad Distrital Francisco José de Caldas, em Bogotá - Colômbia. O objetivo era gerar uma nova perspectiva de profissionalização do ensino, melhorando os sistemas de formação inicial e contínua de professores de ciências. Para conseguir isso, identificamos o conhecimento de conteúdo pedagógico de professores experientes e novatos e monitorados os resultados da implementação da estratégia apoiada por ATLAS.ti ${ }^{\circledR}$ Software para determinar as mudanças nesse conhecimento, estabelecer ligações entre novos professores e especialistas com uma referência desejável e para finalmente trazer a mudança e processos de auto-aprendizagem práticas.

\section{Palavras-chave}

Conhecimento do conteúdo pedagógico, formação inicial, continuada de professores especialistas em educação e novatos, as equipes de trabalho colaborativo

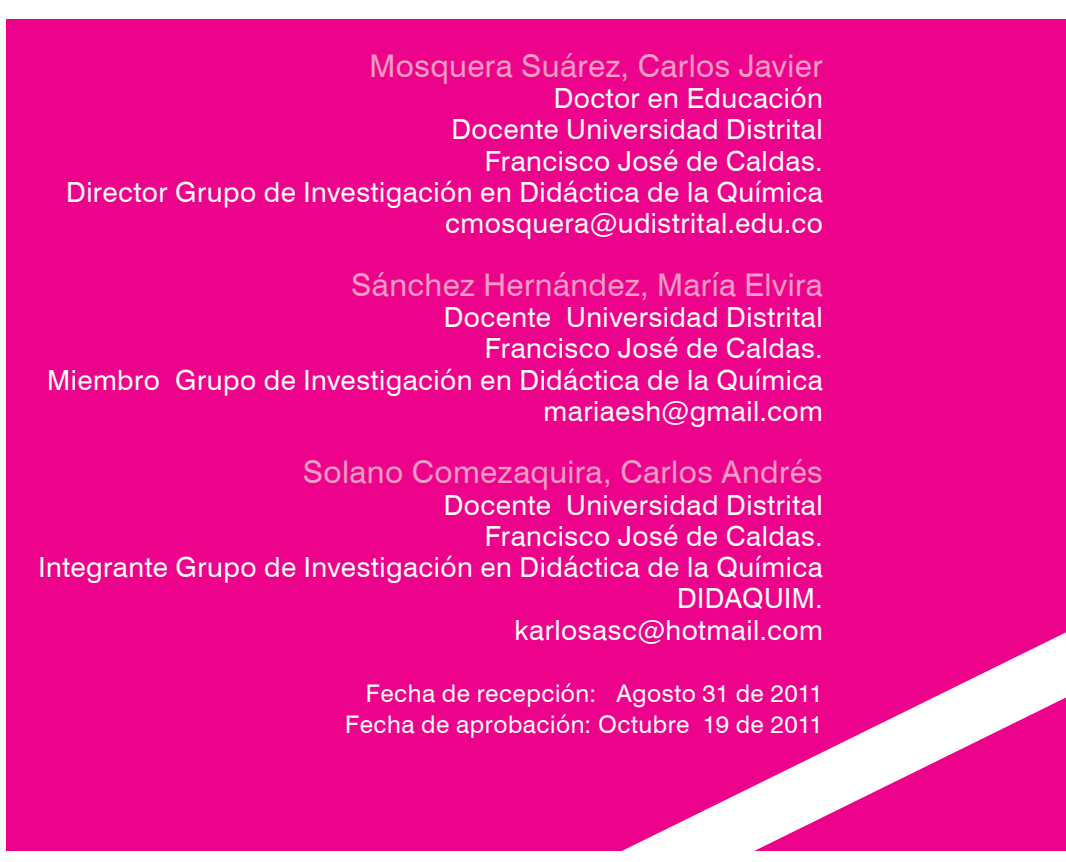



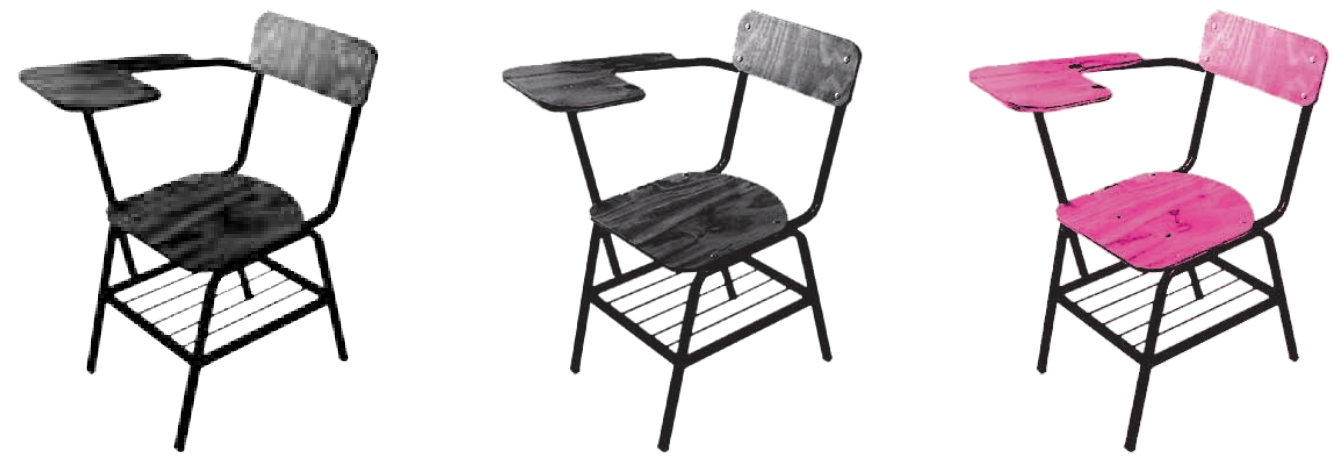

\section{Introducción}

Las fuentes primordiales de renovación de las prácticas escolares se generan en gran medida en las innovaciones que adelantan los profesores tomando como referencia investigaciones contemporáneas en la Didáctica de las Ciencias. Se habla por ejemplo, de la evaluación del currículo, la comprensión de procesos de enseñanza y aprendizaje de las ciencias, la caracterización de estrategias que favorecen el desarrollo del conocimiento científico escolar, y la Formación de Profesores (Mosquera, 2008).

Es este último aspecto sobre el cual se centra la atención de esta investigación, buscando principalmente que el profesor adquiera un enfoque autorregulado y progresivo hacia su ser, saber y saber hacer, enmarcado en revisiones personales que realice sobre las connotaciones propias que tiene de la ciencia, su naturaleza, epistemología, enseñanza y aprendizaje.

La investigación ha buscado que docentes de ciencias nóveles y expertos estén a la vanguardia sociocultural y tecnológica propia de las aulas de clase, y generen actitudes que les permitan vincular las emociones en los sujetos, reconocer las complejidades propias de la escuela y de las políticas educativas, impulsar la participación personal y colectiva de todos los actores de la comunidad educativa, hacer parte de procesos de formación inicial y permanente acompañados de procesos de evaluación coherentes con estas intencionalidades y, finalmente, formarse continuadamente bajo procesos que vinculen la acción, la reflexión y el trabajo cooperativo.

Para dar cuenta de estas perspectivas se hace necesaria la implementación de nuevas estrategias de formación de profesores; particularmente esta investigación se apoya en el modelo epistemológico constructivista y en el modelo didáctico por investigación orientada, de cuya integración se abre el camino hacia el favorecimiento de cambios conceptuales, metodológicos y actitudinales del profesorado hacia la ciencia y hacia la enseñanza de las ciencias.

Las características de estos modelos alternativos de formación de profesores de ciencias tienen en cuenta las ideas, los intereses, las visiones del mundo, las destrezas y actitudes, las experiencias previas, la imagen de la ciencia y de la actividad científica, las necesidades educativas de contextos determinados, así como los propósitos formativos de los profesores que participan en las mismas, entre muchas otras; por ello es necesario iniciar por el reconocimiento explícito de las ideas espontáneas que de su quehacer los profesores puedan manifestar y de igual manera evidenciar la forma de orientación de su enseñanza para comprobar la existencia de alternativas didácticas eficaces que permitan superar los alcances de modelos de enseñanza habituales.

Como lo afirma Mosquera (2009), una investigación desde la Didáctica de las Ciencias sobre formación de profesores, dirigida hacia la consecución de cambios didácticos, no se limita únicamente a que los profesores conozcan nuevos modelos metodológicos o nuevos desarrollos teóricos de las ciencias. Se requiere por el contrario, que el profesorado intervenga activamente y proponga a la luz de nuevas orientaciones disciplinares, prácticas docentes alternativas. Ello implica que el propio profesorado es el que debe identificar y cuestionar de forma argumentada su actividad docente habitual para dar cuenta de las necesidades de cambios. 


\section{Problema de investigación}

En las décadas de los años ochenta y noventa del siglo $X X$, la atención de la investigación didáctica se centró primero en el "conocimiento del profesorado" y, poco después, en lo que "deben saber y saber hacer los profesores" (Gil, 1991) o, de otra forma, en determinar los componentes del conocimiento base que debe tener un profesor para la enseñanza de su disciplina (Shulman, 1987). De este modo, se dio una nueva orientación a la formación del profesorado, en particular en las didácticas específicas destinadas a la formación de profesores de educación secundaria.

La investigación dirigió entonces su mirada al desarrollo del conocimiento profesional del profesorado Abell (2007) $\mathrm{y}$, al mismo tiempo, a la transposición didáctica de los contenidos que conducen a buenas prácticas docentes en la enseñanza de una disciplina Bolívar (2005). Dentro de los contextos educativos actuales, y principalmente en las investigaciones recientes en Didáctica de la Química, un aspecto que cobra gran importancia es el estudio de las concepciones docentes, pues estas son la raíz y fundamento de las acciones que el docente hace explícitas en su práctica educativa.

Como toda persona, el docente convive permanentemente y genera de forma constante y progresiva distintos tipos de creencias, actitudes, conocimientos y saberes, que afectan su desempeño individual, ciudadano y obviamente, el de su actividad escolar cotidiana. Esto lo ubica como un profesional, que fundamentado en su experiencia y conocimientos, genera no solo creencias sino también actitudes hacia las ciencias, y por ende hacia la enseñanza de las ciencias. De otra parte, las implicaciones pedagógicas y didácticas de su actividad o de los procesos permanentes de formación, estimulan reflexiones o actuaciones en la práctica escolar.

Así mismo, para que sean óptimos estos procesos de innovación, y se hagan evidentes cambios didácticos, es indispensable involucrar los resultados de la investigación contemporánea en la Didáctica de las Ciencias, como lo son los trabajos realizados sobre la comprensión de los procesos de enseñanza - aprendizaje, las propuestas de formación de actitudes científicas, el diseño y desarrollo de estrategias que favorezcan al conocimiento científico escolar, la importancia concedida a las concepciones alternativas e ideas previas, la reflexión crítica y la evaluación permanente del currículo, y la reflexión sobre el papel de los problemas socio-ambientales en la didáctica de las ciencias entre otros, que contribuyan a desarollar cambios conceptuales con relación a las nuevas tendencias científicas, epistemológicas y didácticas, sin dejar de lado la implicación de estos cambios en otros simultáneos como lo son los cambios actitudinales y metodológicos en el profesorado de cara al logro de mejores prácticas docentes.

Las concepciones de los profesores le dan sentido a esta práctica, pues la actuación del docente depende de manera directa de sus ideas, creencias, niveles de aceptación o rechazo y formas de decisión. Se establece entonces un puente que vincula el plano cognitivo (actitudes) con el plano cognoscitivo (saberes), dando como resultado unas metodologías de acción, llamadas plano práctico.

A pesar que se tengan claros estos aspectos, la falta de concordancia entre lo que el profesor sabe y todo aquello que hace (relación del plano cognoscitivo con el plano práctico), es evidenciada de forma permanente en la realidad educativa, puesto que se hace a un lado el tercer factor mencionado anteriormente: las actitudes del profesor. Por tal motivo, es importante asumir cambios didácticos que involucren conceptos, actitudes y metodologías de manera constante, simultánea y recíproca.

La propuesta formulada por Lee Shulman (1986), durante la década de los 80's en torno a su presupuesto de Conocimiento Pedagógico del Contenido, PCK, según sus siglas en inglés, generó una corriente de investigación dirigida al estudio de los diferentes tipos de conocimientos que tienen los docentes y sus interacciones, que permiten relacionar las actitudes y saberes, dentro de su práctica profesional. Otros trabajos en educación como los desarrollados por Marcelo (1992) y Mellado (1994), abrieron este campo de estudio en los países hispanos bajo la denominación de Conocimiento Didáctico del Contenido (CDC).

Tomando como referencia estos antecedentes, este trabajo de investigación tuvo como objetivo principal carac- 
terizar el CDC de Profesores de Química en Formación Inicial y en Ejercicio en el marco de espacios académicos de Práctica Profesional en el Programa de Licenciatura en Química de la Universidad Distrital Francisco José de Caldas (Bogotá - Colombia).

A partir del planteamiento del objetivo principal de la investigación y teniendo presentes los procesos de conocimiento y autorregulación que pueden generar los profesores, se ha establecido el siguiente problema de investigación.

¿Cómo se favorece el incremento del conocimiento didáctico de contenido (CDC) a partir del tratamiento de situaciones problema sobre la enseñanza de la química en equipos colaborativos de trabajo de profesores noveles y expertos?
Para dar solución al anterior problema, se establecen tres planteamientos problémicos auxiliares:

¿Cuáles son los CDC previos en Profesores de Química noveles y expertos?

¿Cómo cambia el CDC de los profesores de química noveles a través de su inmersión en el tratamiento de problemas en la enseñanza de la química?

¿Qué tan próximos, luego de la participación en equipos colaborativos, están los CDC de los Profesores de Química noveles y expertos respecto a CDC deseables de referencia?

Para tal efecto, se elaboró un programa de actividades orientado por los directores de esta investigación, el cual tuvo como característica principal delinearse y articularse bajo presupuestos de la epistemología contemporánea sobre las ciencias, concepciones recientes del campo de la didáctica de las ciencias, paradigmas de enseñanza de las ciencias por investigación orientada y actividades de trabajo mediante equipos colaborativos. 


\section{Algunos antecedentes}

Al situarnos en los resultados de los estudios contemporáneos en Didáctica de las Ciencias, es poca la información consignada con relación al Conocimiento Didáctico del Contenido, pues esta se encuentra como una línea de investigación emergente. Sin embargo, para alcanzar el propósito de esta investigación, se agruparon los antecedentes identificados en cuatro grandes grupos: a) Investigaciones Iniciales en PCK, b) Investigaciones Recientes del CDC, c) Investigaciones de CDC en enseñanza de la química, y finalmente, d) Investigaciones en CDC en el Contexto Colombiano.

\section{a) Investigaciones Iniciales en PCK}

Indagar sobre el conocimiento de los profesores como tema no es una preocupación reciente. Grossman, Wilson y Shulman (1989) nos recuerdan que dentro de las investigaciones llevadas a cabo en torno al paradigma perdido, se intentaban establecer relaciones entre el conocimiento del profesor y el rendimiento de los alumnos. Bien es verdad que los investigadores definieron el conocimiento del profesor de forma estrecha, incluyendo desde el número de cursos realizados en su etapa formativa hasta los resultados obtenidos en test estandarizados. Como estos autores afirman, los investigadores llegaban a conocer cuánto conocían los profesores, pero no cómo estaba organizado, justificado o validado ese conocimiento.

Más recientemente, la investigación catalogada en sus orígenes como de "Pensamientos del Profesor", ha abordado desde una perspectiva cognitiva Marcelo (1987); Peterson (1988) el estudio de los procesos de razonamiento, juicio y toma de decisiones que contribuyen al desarrollo de la conducta docente. Esta línea de investigación se ha planteado "explorar la naturaleza, forma, organización y contenido del conocimiento de los profesores" (Grossman, Wilson y Shulman, 1989: 25).

Además, la investigación sobre el pensamiento del profesor ha identificado algunos componentes del conocimiento del profesor: Elbaz (1983) incluye cinco categorías del conocimiento práctico: conocimiento de sí mismo, del contexto, del contenido, del currículo y de la enseñanza. Leinhardt y Smith (1985) categorizan el conocimiento del profesor en conocimiento del contenido y conocimiento de la estructura de la lección. La investigación de "The Knowledge Growth in Teaching" dirigida en la Universidad de Stanford por Shulman (1986) define hasta siete categorías de conocimiento del profesor: conocimiento del contenido, conocimiento pedagógico, conocimiento de currículo, conocimiento de los alumnos y del aprendizaje, conocimiento del contexto, conocimiento didáctico del contenido y conocimiento de filosofía educativa, fines, y objetivos.

El tipo de investigación que generalmente se ha llevado a cabo dentro del paradigma del "Pensamientos del Profesor" se ha centrado más en los aspectos cognitivos que trascienden las particularidades de las materias o asignaturas concretas Wilson y Shulman (1987). Carter (1990) ha clasificado esta línea de investigación en tres grupos. En primer lugar sitúa los Estudios sobre el procesamiento de información y comparación expertos-principiantes. En segundo lugar aparecen los estudios sobre el Conocimiento Práctico que "se refiere de forma amplia al conocimiento que poseen los profesores sobre las situaciones de clase y los dilemas prácticos que se les plantean para llevar a cabo metas educativas en estas situaciones" (Carter, 1990: 299). Por último, Carter sitúa las investigaciones sobre Conocimiento Didáctico del Contenido, para referirse a aquéllos estudios en los que se analiza específicamente el conocimiento que los profesores poseen respecto al contenido que en-

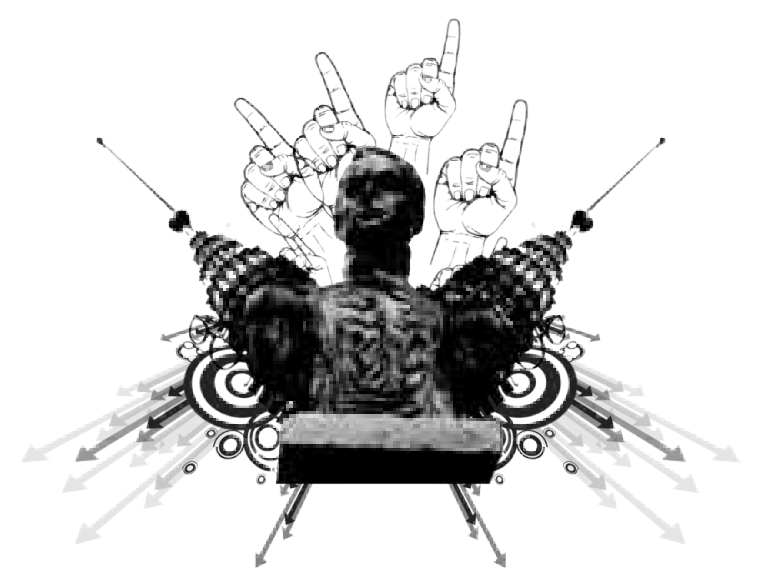


señan, así como la forma como los profesores trasladan ese conocimiento a un tipo de enseñanza que produzca comprensión en los alumnos.

\section{b) Investigaciones Recientes del CDC}

Los trabajos relativos al CDC están aumentando mucho en los últimos años Garritz (2006) y el CDC muestra gran vitalidad en la actualidad. No obstante, todavía es necesaria mucha más investigación sobre el CDC en determinados temas de ciencias y sobre el propio CDC como modelo en sí mismo. Al igual que sucede en otras didácticas específicas, el CDC se usa en didáctica de las ciencias, por un lado, como modelo para describir e interpretar el modo en que los profesores en formación inicial y principiantes, aprenden a interpretar y transformar el contenido de un tema en significados comprensibles para un grupo de estudiantes en el aula Van Driel, Verloop y De Vos (1998).

Por otro lado, aunque el CDC parece ser idiosincrásico de cada profesor, varias investigaciones sobre el CDC de profesores de ciencias defienden la posibilidad de hacer algunas generalizaciones potencialmente útiles para la formación de otros profesores de ciencias Loughran, Berry y Mulhall (2006). Desde el punto de vista del diseño de una investigación, se han sugerido distintos procedimientos, métodos e instrumentos de recogida de datos para intentar sacar a la luz y describir el CDC del profesorado. Baxter y Lederman (1999) los clasifican en tres tipos principales: Procedimientos inferenciales y convergentes, por ejemplo cuestionarios de elección múltiple y abiertos; mapas conceptuales y representaciones gráficas; y evaluaciones por métodos múltiples Van Driel y De Jong (2001).

\section{c) Investigaciones del CDC en la Enseñanza de la Química}

Respecto al CDC en la enseñanza de la química se han encontrado relativamente pocos estudios, dentro de los que podemos mencionar los siguientes:

Clermont, Krajcik y Borko (1993), realizan una exploración de la naturaleza del crecimiento del CDC que ocurre a profesores de ciencias de nivel medio que participan en un taller intensivo de capacitación sobre enseñanza usando demostraciones para dos conceptos básicos en física y química: la densidad y la presión del aire. Estos autores encuentran que el CDC de los profesores de ciencias puede crecer a través de talleres intensivos orientados a desarrollar habilidades. Sin embargo, aunque hubo un crecimiento en los repertorios representacional y adaptacional de estos profesores, en otros dos aspectos del CDC parece haber ocurrido mucho menos avance; esto es, en el conocimiento asociado con la evaluación crítica y del contenido y con la selección instruccional. Estos hallazgos indican que el CDC es un sistema de conocimiento complejo y sugieren que sus diferentes componentes pueden mostrar distintas velocidades de crecimiento en una actividad de capacitación.

Por otra parte Clermont, Borko y Krajcik (1994), examinan el CDC de profesores de química, tanto con experiencia como principiantes, que usan como estrategia la enseñanza por demostraciones, ya que ésta se considera un componente importante del repertorio pedagógico de los profesores de ciencias y es un área que no está bien desarrollada. Los hallazgos sugieren que los profesores con experiencia, comparados con los nóveles, poseen un mejor repertorio adaptacional y representacional para la enseñanza de conceptos fundamentales en química. También parecen ser más conocedores de la complejidad de las demostraciones químicas, cómo dicha complejidad puede interferir con el aprendizaje y cómo las demostraciones químicas más simples pueden promover mejor el aprendizaje de conceptos.

Veal (1998), realiza un estudio sobre la evolución del CDC de futuros profesores de química de secundaria sobre aspectos de termodinámica y sus resultados se resumen de la siguiente manera:

Los futuros profesores desarrollan diferentes tipos de CDC: general, de dominio específico y de tópico específico, los cuales difieren en sus propósitos, usos y aplicaciones Veal y Makinster (1999); la velocidad y el grado de desarrollo de cada uno de estos tipos de CDC se encuentra en función de su formación y experiencia anterior.

- El desarrollo del CDC de tópico específico ocurrió antes del de dominio específico.

- Los profesores en formción inicial demostraron y desarrollaron un entendimiento fundamental de la enseñanza y el aprendizaje de las ciencias que servirá 
como base para el desarrollo de un CDC de dominio específico mayor.

Van Driel, Verloop y de Vos (1998), realizan un estudio empírico enfocado al CDC de un tópico específico, el equilibrio químico; allí incluyen, además, una revisión de la literatura sobre el CDC de los profesores con respecto a la enseñanza en general y en el dominio de la educación en ciencias. Encuentran que las estrategias de enseñanza identificadas en el estudio no son útiles en un sentido universal, sino se refieren exclusivamente al tópico involucrado; aún más, como los profesores enseñan tópicos específicos, estas estrategias adicionan un elemento único y valioso al conocimiento básico educacional.

Dawkins y Butler (2001), analizan el CDC de siete estudiantes del profesorado de ciencias del segundo año universitario respecto al concepto de mol. Encuentran que las estrategias empleadas por ellos para la enseñanza tienen marcada influencia de los libros de texto de química, en los cuales no siempre se manejan los conceptos como los manejan los científicos (no usan, por ejemplo, el término "cantidad de sustancia"). Así mismo, hallan que un entendimiento claro del concepto no necesariamente implica que se usen las estrategias más adecuadas para la resolución de problemas relativos a la proporción entre masa y moles.

De Jong, Veal y Van Driel (2005), realizan una recopilación de los estudios llevados a cabo con un enfoque sobre el conocimiento básico de los profesores de química, centrándose sobre el Conocimiento del Contenido (CC) y el CDC, esto es, los dos tipos de conocimiento que están determinados por la naturaleza del tópico específico enseñado. Estos autores resumen la variedad de aspectos del CDC de los profesores de química de la siguiente manera:

- Los profesores de química con insuficiente CDC de tópicos específicos pueden, en ocasiones, realizar demostraciones que pueden reforzar las concepciones alternativas de los estudiantes.

- Un CDC cercano al deseable, implica el conocimiento de cómo aprenden los estudiantes y el reconocimiento de representaciones alternativas, como requisitos para la selección y uso de explicaciones analógicas apropiadas y efectivas.
- La selección, por parte de los profesores de química, de una estrategia para la enseñanza de cálculos estequiométricos, con frecuencia no es muy adecuada desde la perspectiva del aprendizaje del estudiante.

Un trabajo reciente sobre la enseñanza de las ciencias en el bachillerato lo desarrollan Treagust y Mamiala (2003), donde analizan, con ejemplos, los cinco tipos de explicaciones que emplean los profesores durante sus clases introductorias de fisicoquímica y de química orgánica, acerca de los tres niveles de representación usados en la química: el macroscópico, el submicroscópico y el simbólico. Dichas explicaciones identificadas fueron:

- Analógicas: un fenómeno o experiencia familiar se emplea para explicar algo poco familiar.

- Antropomórficas: a un fenómeno se le dan características humanas para hacerlo más familiar.

- Relacionales: una explicación que es relevante dada las experiencias personales de los aprendices.

- Basadas en problemas: una explicación demostrada a través de la resolución de algún problema.

- Basadas en modelos: utilizar un modelo científico para explicar un fenómeno.

Talanquer (2003), ha trabajado vigorosamente con sus colaboradores en la Universidad de Arizona para poner al punto los cursos de formación de profesores para la educación en ciencias. En este equipo se han incorporado cursos con el nombre de "Métodos de Enseñanza del contenido" que versan precisamente sobre el CDC específico del área de concentración del futuro profesor (biología, química, física o ciencias de la tierra).

\section{d) Investigaciones en CDC: Contexto Colombiano}

Dentro del contexto colombiano, las Facultades de Educación en Ciencias, han ido desarrollando algunos proyectos de investigación en relación con el Conocimiento Didáctico del Contenido. Los antecedentes en Colombia identificados y más representativos son:

El estudio realizado por Ariza y Parga (2008) sobre el conocimiento didáctico del contenido de los profesores de química en relación con la combustión. En este, se considera en primer lugar la importancia de la selección y secuenciación del contenido a enseñar por parte del profesor con relación a la historia y la epistemo- 
logía de la química, para diseñar lo que Mora y Parga (2007) han denominado Tramas histórico/epistemológicas (THE) como componentes intermediarios del diseño curricular. Para el caso particular de la combustión se tiene en cuenta la rivalidad de las teorías del flogisto y del oxígeno. Posteriormente, se preguntan sobre el conocimiento histórico y epistemológico del profesorado, sobre el valor e importancia que le dan, considerando también los contenidos metacientíficos o metadisciplinares (propios de la historia y la epistemología de la química) en el diseño curricular antes hablado, en donde los profesores podrán explicitar, comunicar y estructurar su pensamiento acerca de la ciencia antes de ser enseñado.

Pero la THE tendrá sentido al articularse a una trama de contenido (que relaciona los conocimientos psicológicos del aprendizaje y del contexto) no a la manera de un concepto estructurante que requiere de un alto nivel de inclusividad y abstracción para ser llamado estructurante Aduriz, Izquierdo y Estany (2002), sino de una trama de conceptos relacionados con la combustión. Los autores afirman, que se considera la trama de la combustión porque está relacionado con la química, con la biología y la física, así como con contextos sociales, técnicos y cotidianos (los que se podrían llamar relaciones CTS); en tal sentido, se hace interdisciplinar, al igual que para la comprensión de los estudiantes podrá facilitar el proceso cognitivo de ellos al relacionarlo no solamente con fenómenos científicos, sino que le permite ser analizados desde problemas sociales (por ejemplo la combustión y los problemas ambientales).

A partir del análisis de los THE establecidos por los profesores de química presentes en el estudio, Ariza y Parga concluyen que es necesaria la investigación del conocimiento didáctico del contenido curricular en química, si se quiere mejorar la calidad de la enseñanza y la profesionalización docente; además, que las THE y las Tramas de contenido evidencian la construcción de un CDC en el profesorado y con ellas se podrá mejorar la comprensión en el estudiantado de temas que por lo general se abordan en forma aislada, descontextualizadas y sin sentido.

El Conocimiento Didáctico del Contenido en Química: integración de las Tramas de contenido / histórico - epistemológicas con las Tramas de Contexto / Aprendizaje. Mora y Parga (2007). A partir del análisis del contenido de diversas fuentes de información con relación a las tramas de contenido, tramas de contexto y CDC, Mora y Parga, muestran una panorámica general y un marco conceptual de un campo disciplinar reciente que puede contribuir al desarrollo profesional del ejercicio docente en química con distintos indicadores explicitados desde los trabajos de varios grupos centrados en el desarrollo de gradientes evolutivos de las ideas del estudiantado y profesorado.

\section{Concepción y estrategia metodológica seguida}

La investigación en Educación, como en cualquier otro campo del saber, tiene como principal finalidad la de colaborar a la indagación y crítica sistemática de los saberes adquiridos, avanzando así en el desarrollo del cuerpo teórico que la sustenta. En el intento por comprender la realidad actual de la formación de profesores y los fundamentos teóricos promovidos desde la Práctica Profesional Docente de Licenciatura en Química, la presente investigación se plantea como el estudio de la singularidad del programa de formación inicial de profesores de secundaria para la enseñanza de la química, a partir de la observación y participación en el desarrollo de las prácticas llevadas a cabo por un grupo de docentes en formación inicial.

El seguimiento y análisis de los programas de formación inicial del profesorado ha sido desarrollado desde la perspectiva de una investigación que se inscribe en el campo de la metodología cualitativa. Sobre la base de un diseño interpretativo se llevó a cabo un estudio de caso en dos contextos de formación de profesores específicos: la formación inicial, que alude a los profesores nóveles y la formación continuada, referida a los profesores expertos.

A través de la integración en un contexto de equipos colaborativos de trabajo se comprobó la validez del supuesto originado a partir de esta investigación: Los CDC de profesores de química en formación inicial y de profesores de química en ejercicio, pueden fortalecerse conceptual y actitudinalmente a través de la inmersión en problemas sobre el aprendizaje de la química que se resuelven y se desarrollan en la práctica educativa mediante equipos colaborativos de trabajo. 
Todo esto sin perder de vista lo que se espera de una investigación cualitativa interpretativa: "descripciones abiertas", "comprensión mediante la experiencia" y "el estudio de los actos y los significados que los actores adscriben a lo que sucede en un contexto social particular" (Stake, 1998).

Por medio de la observación, la participación y la interpretación de una realidad educativa concreta y compleja, como es la formación de profesores, nos acercamos a la comprensión de las exigencias que la sociedad, a nivel global, y el sistema educativo, a nivel más concreto, nos plantean en relación con la formación inicial y permanente de los futuros y actuales profesores de química, en el marco de la Práctica Profesional Docente.

Se pretende alcanzar un equilibrio teórico y práctico entre profesores expertos y noveles, de modo que el profesor experto reconozca tendencias actuales de la investigación en educación en ciencias y el profesor novel incremente estrategias prácticas de trabajo en el aula. Todo esto enmarcado en los parámetros que se obtienen al caracterizar el Conocimiento Didáctico del Contenido (CDC) en términos de cambios didácticos, buscando mejoras en las prácticas educativas en los profesores de ciencia.

La puesta en marcha de este proyecto se planteó mediante el desarrollo de un programa de actividades sustentado en tres variables: variable interviniente; propuesta de investigación por equipos colaborativos de trabajo, Variable dependiente; cambios didácticos en los profesores noveles y expertos, Variable independiente: las actividades del profesor. El programa fue diseñado bajo el modelo de enseñanza de las ciencias por investigación orientada recurriendo a trabajos colaborativos de grupos de profesores. Para desarrollar esta problemática, se han considerado dos aspectos - supuestos - con relación a los docentes de Química expertos y noveles: En primer lugar ¿Qué se debe hacer en el aula? Y en segundo lugar ¿Cómo se debe enseñar ciencias? En esta investigación se definieron tres componentes fundamentales del CDC que son tomados como nuestras categorías de análisis: a) Imagen de ciencia b) Relación enseñanza - aprendizaje de la ciencia e c) Implicaciones del contexto escolar. Estas categorías se convierten entonces en los ejes que direccionan la metodología de este trabajo, cada uno analizado a partir del ser, saber y saber hacer del profesor (Ver Cuadro 1).
El proyecto se desarrolla en un estudio longitudinal para lo cual se tiene en cuenta la identificación de las ideas previas de los docentes a partir de un instrumento por Diferencial Semántico, la grabación en audio y en video de las sesiones en los equipos colaborativos y de las actividades docente y finalmente, el estudio de posturas finales sobre las ciencias y la enseñanza de las ciencias indagadas a través de entrevistas y cuestionarios tipo Liker. Los estudios sobre las elaboraciones en equipos colaborativos y las producciones en las actividades docentes, se estudiaron mediante el Software de Análisis de Datos ATLAS.ti.

\section{Análisis inicial: \\ Instrumento por diferencial semántico}

Se diseñó, validó y aplicó un instrumento por diferencial semántico, dirigido a profesores noveles y expertos sobre su quehacer en el aula de clase. El objetivo principal fue el de identificar las actitudes hacia la labor docente y caracterizar los CDC previos de profesores de Química noveles y expertos, a partir de la postura tomada por cada uno de ellos frente a las sentencias presentadas. Cabe resaltar que dichas sentencias se plantean en relación directa con las categorías e indicadores propuestos para la presente investigación.

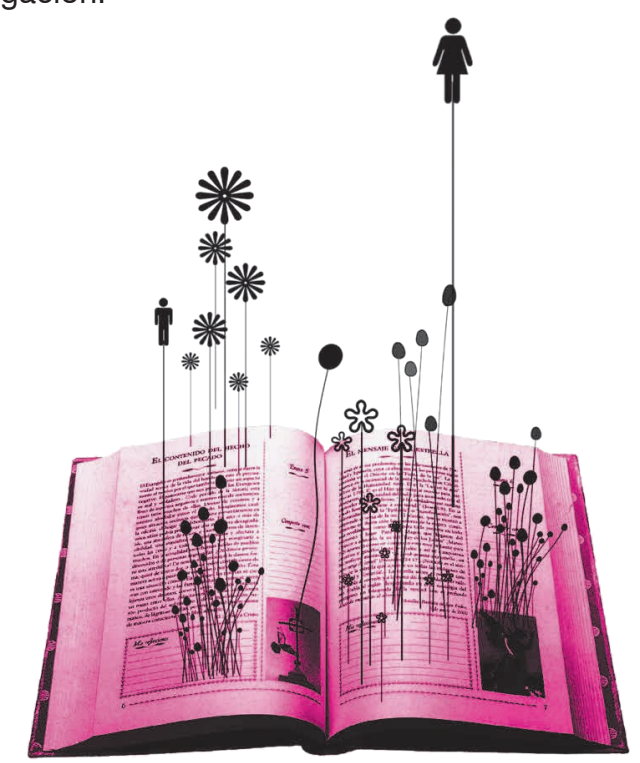


Análisis permanente (a):

Observaciones de Clase - Grabaciones

Un primer acercamiento para comprender la realidad formativa del profesor en formación inicial y en ejercicio fue conocer como se tratan los contenidos programados y percibir el ambiente y la dinámica del proceso de aula y detectar igualmente las situaciones problema presentadas en la enseñanza de la química.

Cuadro No. 1
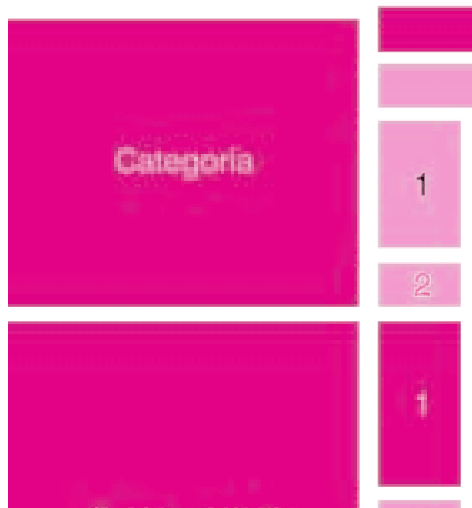

Enseñanza Y

Aprendizajo

De La Ciencia
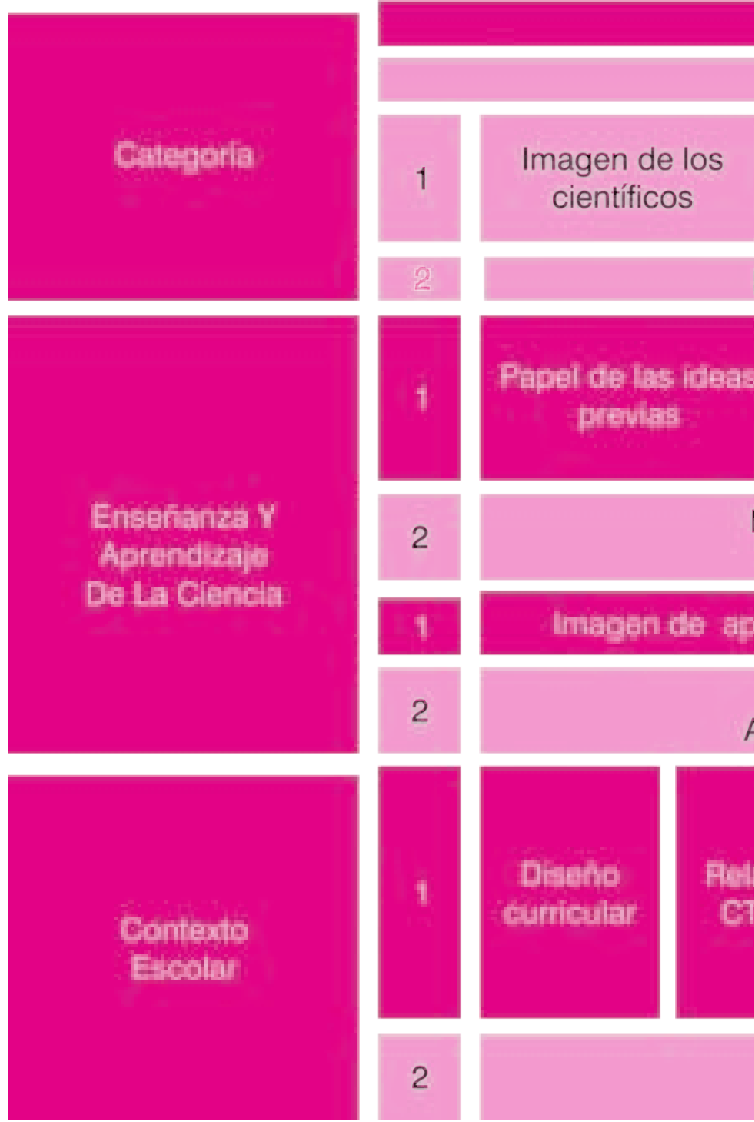

Imagen de aprendizaje
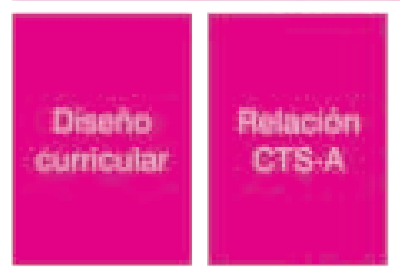

Para tal efecto se hicieron registros en video para analizar el quehacer de cada uno de los docentes. Estos, inmersos en sus trabajos cotidianos, actúan de acuerdo a sus concepciones y creencias. Se categorizan cada una de las clases grabadas y se determina el acercamiento gradual hacia posturas y prácticas innovadoras en la enseñanza de la Química. Estas se analizaron a través del Software de análisis de datos ATLAS.ti.

Indicadores (1)

Consecuencias Contratables (2)

Imagen de Imagen de actividad científica progreso de la ciencia

Paralelo "ciencia convencional" y "otras ciencias"
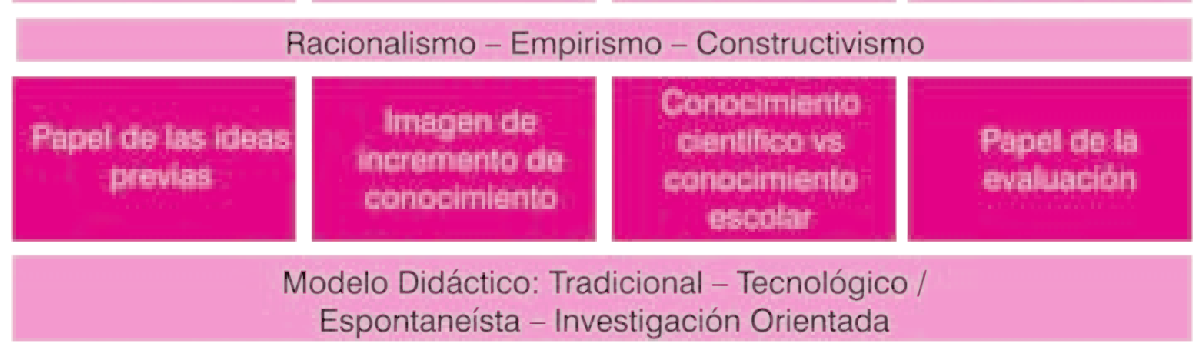

Imagen interdisciplinaria de los conocimientos
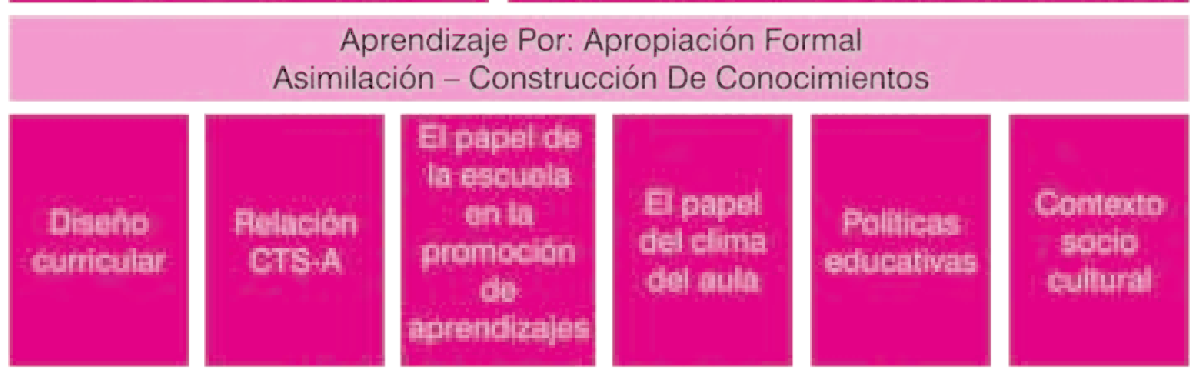

Producto Formal - Proceso Tecnológico /

Espontaneísta - Complejo 


\section{Análisis permanente (b): Equipos colaborativos de trabajo Análisis cualitativo mediante el uso del Software ATLAS.ti.}

La estrecha relación existente entre las actitudes y los saberes (planos cognitivo y cognoscitivo) es la que finalmente determina las acciones y hechos durante la práctica docente. El docente actúa a partir de sus preconceptos, concepciones, objetivos personales, creencias, entre otros muchos factores, y finalmente la suma de todas estas variables dan características particulares a la acción docente.

Generar espacios para el tratamiento de situaciones problema sobre la enseñanza de la química en equipos colaborativos de profesores noveles y expertos, es la parte trascendental que produce los cambios actitudinales, conceptuales y procedimentales en el grupo de docentes con el que se trabajó. En otras palabras, estos cambios son los que en suma, podemos considerar como cambios didácticos que representan las variaciones en el CDC de los profesores. Es por ello que la variable interviniente ha sido un programa de actividades (elaborado por los autores de esta investigación) en el que se abordaron las situaciones problemas en la enseñanza de la química.

Para el desarrollo de las actividades dentro de los equipos colaborativos de trabajo, se establecieron unos parámetros de seguimiento.

1. Identificar los problemas cotidianos existentes en las Clases de Química.

2. Verbalizar estos problemas dentro de los equipos colaborativos de trabajo.

3. Exposiciones, presentaciones y talleres dirigidos por parte del grupo de investigación para abordar los problemas tratados.

4. Complementación por parte de todo el equipo colaborativo de trabajo, por medio de la realización de lecturas de documentos seleccionados.

5. Sugerir a los profesores noveles y expertos que incluyan los nuevos conocimientos a sus prácticas. La implementación o no de estas sugerencias, es analizada a través de grabaciones de las actividades académicas - explicacio- nes, talleres, evaluaciones, laboratorios, etc. Finalmente, aplicada una entrevista de cierre y sus resultados complementados en la aplicación de un cuestionario escala Liker, permitió dar cuenta de los cambios didácticos logrados, los cuales en suma, en el contexto de esta investigación, nos dan luces para identificar el CDC de cada uno de los agentes participantes en este trabajo. Por efectos de espacio, únicamente se presentan a continuación los análisis logrados para uno de los docentes nóveles participantes.

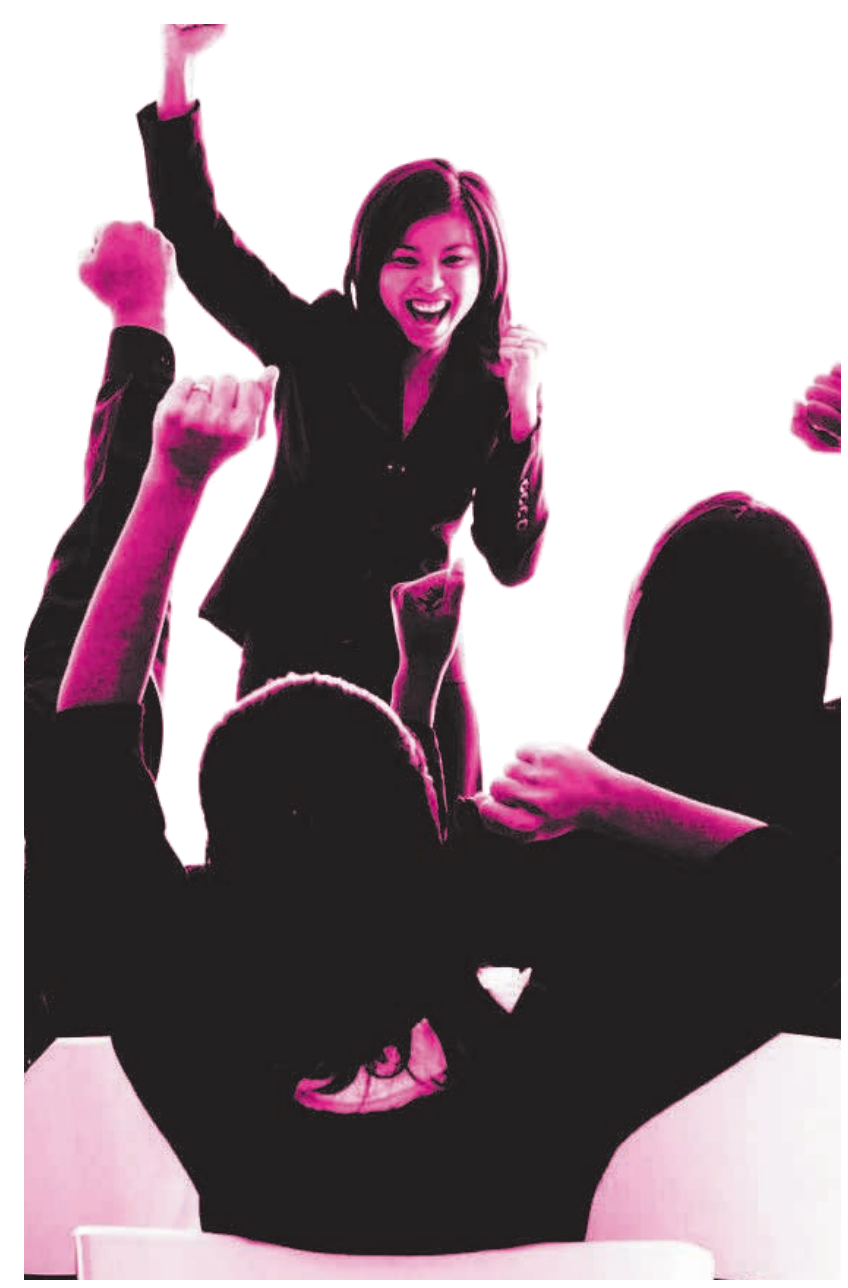




\section{Resultados obtenidos}

\section{El caso de "Felipe"1 : un cambio didáctico en la formación inicial}

Para satisfacer las necesidades de esta investigación, se trabajó con el grupo de Práctica Profesional Docente (2011 - I) del Proyecto Curricular de Licenciatura en Química de la Universidad Distrital Francisco José de Caldas (Bogotá - Colombia), que contaba con seis (6) docentes nóveles y dos (2) docentes expertos. Para este artículo, resaltamos los resultados obtenidos en uno de los docentes nóveles con el que trabajamos.

Con relación a la imagen de ciencia, inicialmente, presentó una visión multivalente frente a esta categoría que no brinda la opción para categorizarlo, pues manifiesta opiniones contradictorias frente a una única concepción de ciencia. Se marcaba una ligera tendencia empirista en cuanto a que demostraba un cierto nivel de aceptación a la visión convencional del método científico; además, reconoció que la ciencia no se desarrolla en etapas plenamente delimitadas y que también es necesaria la teorización para el aprendizaje de las ciencias. Durante sus clases y sus prácticas de laboratorio, mostraba permanentemente una visión rígida exacta e infalible, socialmente descontextualizada. Además, por lo generar no hacía énfasis en el trabajo de los científicos ni en los procesos de crecimiento de las ciencias; sumado a esto, no evidenciaba una ciencia articulada, sino que por el contrario deja entrever una imagen de la ciencia lineal, aproblémica y acumulativa en la que muy poco involucra la historia y la epistemología del conocimiento científico tratado en clase.

Al referirnos a la enseñanza de las ciencias su visión inicial estuvo direccionada principalmente por el modelo espontaneísta: desde este modelo planteaba la importancia de involucrar al estudiante en su proceso de formación, desde el convencimiento que el contenido verdaderamente importante para ser aprendido por ese alumno ha de ser expresión de sus intereses y experiencias y se halla en el entorno en que vive. Sin embargo, desde esta visión se desvirtúa el papel de los aprendizajes previos que posee el estudiante. Consecuentemente según esta visión el estudiante construirá su conocimiento escolar basándose exclusivamente en su interpretación del conocimiento científico. En sus prácticas de enseñanza situaba su postura en un modelo tradicional, ya que en ningún momento se interesa por conocer las ideas previas o concepciones alternativas de sus estudiantes. Comenzaba la explicación de un nuevo concepto para sus estudiantes sin aproximarlos a algunos referentes históricos o socioculturales del mismo. Al basarnos en el hecho que es un concepto nuevo, no proporciona un ambiente para que el estudiante se acerque a su comprensión; en cambio, asume una visión exclusivamente algorítmica del concepto, para este caso "densidad". Se infiere a partir del comportamiento de Felipe, que intentaba acumular una serie de conceptos y temas. Finalmente es totalmente evidente, que asocia el término "evaluación" a una simple calificación sancionatoria. La usa a manera de "represalia" o "castigo" para los estudiantes que no obedecen sus indicaciones, más que una forma de motivar y retroalimentar los contenidos en química.

Las concepciones iniciales de Felipe con relación al aprendizaje de las ciencias es consecuente con el modelo de asimilación, ya que solo puso de manifiesto la necesidad de llevar los contenidos a un contexto próximo al contexto real del estudiante, sin generar espacios para que el propio estudiante cree sus conocimientos. Esta postura se hizo de nuevo evidente cuando al hablar de la diversidad de los estudiantes y de sus procesos de aprendizaje. En cuanto a las características y finalidades de la evaluación no evidenciaba una postura definida, pues sus concepciones era difusas entre los modelos tecnológico, espontaneísta e investigación orientada, con lo cual pone en manifiesto que para un momento inicial no tenía claridad en sus concepciones; veía la evaluación únicamente como el instrumento final y sancionatorio de un contenido temático desarrollado.

Consecuentemente con sus prácticas tradicionales de enseñanza, deja a la luz una visión formal de aprendizaje de los conocimientos científicos por parte de los estudiantes, según esto y en acuerdo con lo expuesto por Por- 
lán (1989: 337) "concibe el hecho de aprender como un acto de apropiación cognitiva, mediante el cual, el sujeto que aprende, toma del exterior, ya sea de otra persona de un texto escrito o de la propia realidad, unos determinados significados. Presupone que la comunicación de significados es un proceso neutro y objetivo donde los mensajes no sufren alteraciones ni deformaciones en el proceso que va desde el sujeto que emite al sujeto que recibe. Presupone también que de cada concepto, proceso o dato, que es conveniente enseñar y aprender, sólo existe un único significado correcto. El que va a aprender algo lo hace porque, o no posee dicho significado, o el que posee es incorrecto. Esta idea sobre el aprendizaje ha sido representada por las metáforas del vaso vacío o de la mente en blanco".

Finalmente al referirnos a las observaciones iniciales, en cuanto al contexto escolar inicialmente, su postura no estaba completamente definida con relación al contexto escolar, pues presentaba rasgos formales y espontaneístas, al afirmar que únicamente se puede aprender ciencias si los temas están perfectamente organizados (acumulativos) y al reconocer tan solo una parte del la interacción enseñanza - aprendizaje en los contextos escolares en el aula, respectivamente, presenta una ligera inclinación al enfoque complejo, al aceptar métodos alternativos como la resolución de problemas y reconocer la diversidad del aula para generar actitudes favorables hacia la química. No consideraba importante reconocer la diversidad en el aula de clase ni tampoco considera su actitud dentro de la misma como una posibilidad o no de aprendizaje, y la evaluación sigue siendo un mecanismo de represión. La evidencia demostró que Felipe no tomaba en cuenta significativamente los contextos sociales, culturales y económicos de sus estudiantes. Su interés se centra primordialmente en el conocimiento científico dejando de lado conocimientos complementarios de gran valor en la enseñanza de las ciencias; es el caso de la relación CTS-A, que se tuvo en cuenta superficialmente y que no logro trascender en la clase. Por otra parte nunca realizaron actividades para reconocer la diversidad de población existente.

Con el transcurrir del tiempo y su inmersión en las charlas en equipos colaborativos de trabajo, la imagen de las ciencias que mostró en sus clases fue cambiando positivamente, al empezar a incluir referentes históricos de los conceptos tratados en clase, además de dejar ver a sus estudiantes que muchas teorías científicas no son solo el resultado de la sumatoria de teorías anteriores y que por el contrario se han producido rupturas y confrontaciones de ideas entre diferentes comunidades científicas; esto permitió que la imagen de ciencia comunicada a los estudiantes se transformara de aproblémica y ahistórica en algo completamente diferente. Además de esto empezó a crear puentes entre los conocimientos científicos estudiados en clase y la cotidianidad de los estudiantes.

La forma de enseñar de Felipe se enriqueció al incluir el estudio de ideas previas de los estudiantes, generar procesos de autorregulación y ampliar la visión de la evaluación convirtiéndola en un proceso permanente y formador. Su visión de los procesos de aprendizaje llevados a cabo por los estudiantes se sustentó principalmente desde el modelo de construcción de conocimiento, con lo que se puede ver que después de la inmersión en los equipos colaborativos de trabajo Felipe concibe el aprendizaje como un proceso complejo y no como una simple memorización de conceptos. Desde esta perspectiva infiere que los procesos de aprendizaje de las ciencias que involucran las concepciones preexistentes, las practicas experimentales y la interpretación que hacen los estudiantes de la información presentada por el docente y por otras fuentes son un buen camino para lograr un aprendizaje científico relacionado con la realidad del estudiante y capaz de crear destrezas para resolver problemas de la vida cotidiana.

Considera importante el contacto con la realidad para el aprendizaje científico escolar; de igual manera, afirma que el aprendizaje científico es significativo cuando el alumno tiene un interés personal relacionado con lo que aprende. Entra en pequeñas contradicciones, al no tener claro las actuaciones del profesor frente a la diversidad socioeconómica y las diferencias de ritmos de aprendizaje de sus estudiantes, a pesar de esto el hecho de identificar una diversidad tanto socioeconómica como en ritmos de aprendizaje es un cambio positivo que conlleva a ver las relaciones dentro del aula como un factor importante en la enseñanza y el aprendizaje de las ciencias.

Es evidente que el trabajo en equipos colaborativos con otros docentes le permitió a Felipe mejorar sus prácticas educativas, en primera instancia es de resaltar que logro generar una reflexión autorregulada de sus actividades y a partir de eso reconocer errores para mejorarlos y potenciar y acrecentar las virtudes que ya se tenían. Por otra parte el tratamiento de un programa de actividades dirigi- 
do por los investigadores dio pautas y herramientas para iniciar un cambio en pro de acercar su CDC y acercarlo a un deseable de referencia.

Esta investigación no tiene como propósito mostrar lo "bueno" o "malo" del conocimiento de los profesores, sino más bien, comprender los conocimientos disciplinares, pedagógicos y didácticos que poseen, explicar los aspectos que están asociados a su construcción, integración y especialmente incremento, así como identificar diferentes características propias de sus actitudes y cómo estas son integradas y de qué manera influyen en los procesos de enseñanza y aprendizaje de las ciencias. Por consiguiente, de manera general, las características del CDC y el incremento producido a partir de nuestra actuación como investigadores se presentan a continuación.

\section{Resultados generales}

Anteriormente se presentaron en detalle los resultados de un docente en formación inicial que ha participado en esta investigación; a continuación se presentan de forma resumida los resultados generales del grupo de docentes:

\section{Antes de la inmersión en los equipos colaborativo de trabajo}

Con respecto a las ciencias los docentes noveles no generan una postura definida, sin embargo, la tendencia con mayor aceptación es el empirismo, reconociendo que la construcción y evolución de la ciencia se ha dado fundamentalmente por la observación directa de la realidad y la experimentación. Sin embargo, estas concepciones empiristas no son arraigadas y más bien son respuestas espontáneas o reflejos inmediatos a las cuestiones planteadas por el instrumento.

El docente experto muestra una marcada tendencia al empirismo, algo similar al grupo de noveles pero a diferencia del resto de docentes él demuestra que ha forjado esta postura desde su amplia experiencia docente.

En lo referente a los procesos de enseñanza - aprendizaje el grupo en general (docentes noveles y docente experto) sustentan una postura acorde con el modelo de enseñanza por investigación orientada y de aprendizaje por construcción de conocimiento; según esto, identifican la complejidad de la relación docente - estudiante - conocimiento y las múltiples variables que en esta intervienen. Relación que según Porlán (1998) en cuanto a la enseñanza debe incluir las dimensiones relativizadoras, complejas e investigativas que se sitúan entre las concepciones crítica e interpretativa de la teoría de la enseñanza; y que en cuanto al aprendizaje, lo ve un proceso en que el individuo y el grupo no sólo desarrollan gradual y progresivamente su particular estructura de significados, sino que, precisamente por ser un proceso en el que el sujeto elabora los significados, y no simplemente los toma o asimila, también construyen singularmente el camino específico de su evolución.

En la categoría de contexto escolar al igual que en la referente a la imagen de ciencia todo el grupo de docentes (noveles y el docente experto) no se decide por una única postura y aunque todos se inclinan ligeramente por el enfoque complejo se puede leer que en realidad algunos campos de lo catalogado como contexto escolar en esta investigación no juegan un papel decisivo e incluso algunos no son tomados en cuenta en la labor docente de este grupo de profesores noveles.

\section{Después de la inmersión en los equipos colaborativo de trabajo}

En términos generales los docentes transformaron sus posturas pasando de un empirismo moderado a una visión constructivista de las ciencias, pues identificaron características que convierten la actividad científica en una actividad social, humanizada y desarrollada en contextos sociales y culturales. Se alejan así de la visión clásica del científico como un ser sobrenatural y con unas características especiales que muy pocos pueden llegar a alcanzar. Sus posturas renovadas frente a las ciencias las dejaron ver en las clases que orientaron articulando el conocimiento científico con el contexto histórico en el que se desarrollaron y proponen tener en cuenta el contexto socio-cultural del estudiante a la hora de desarrollar las clases.

El docente experto reconoció algunas posturas empiristas en su quehacer en el aula y generó cambios al respecto apoyando las iniciativas de los docentes noveles de incluir factores como la historia de las ciencias y el cambio 


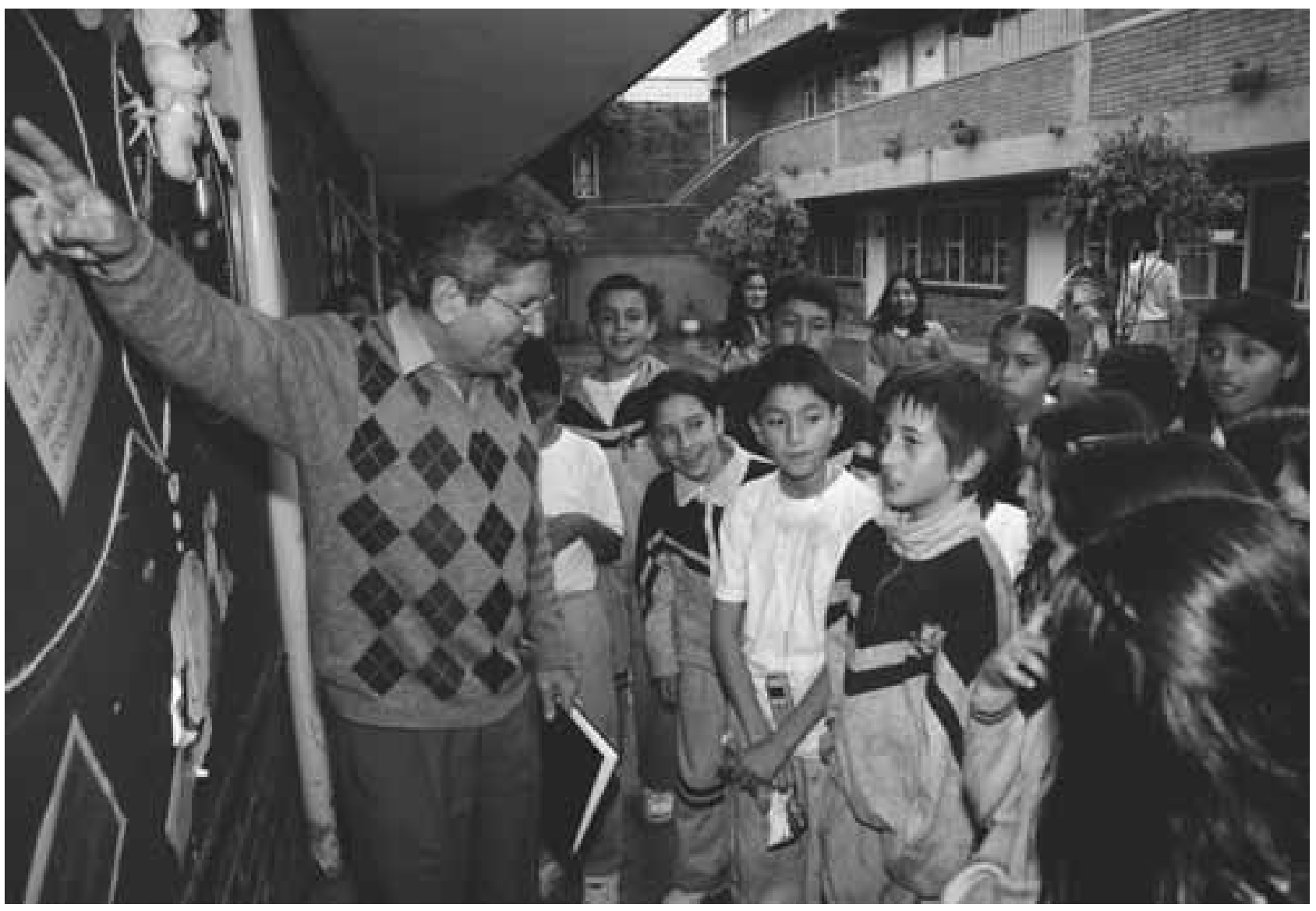

a un verdadero aprendizaje significativo contextualizando socio-culturalmente los conceptos a desarrollar en clase.

Aun cuando la visión inicial del grupo de docentes en general era tendiente a la enseñanza por investigación orientada y al aprendizaje por construcción de conocimientos; después de la inmersión en los equipos colaborativos se avanzó en la identificación y utilización de las ideas previas que poseen los estudiantes, así mismo como ya se mencionó, los conceptos desarrollados fueron impregnados con referentes históricos. Simultáneamente se dejó de ver el aprendizaje como la simple memorización de conceptos y se intentó identificar como un proceso complejo, en este sentido los docentes noveles principalmente empezaron a proponer los ejercicios trabajados en clase como situaciones problemas en contexto que retaran los conocimientos de los estudiantes y que no fueran la aplicación de un simple algoritmo.

Algunos componentes del contexto escolar inicialmente no jugaban un papel importante en las actividades llevadas a cabo por los docentes noveles, sin embargo, después de la inmersión en los equipos colaborativos de trabajo se reconoció que es necesario tener un buen clima de aula, una planificación eficaz y contar con los aportes de los estudiantes en los objetivos a desarrollar en cada unidad y en el proceso evaluativo de las actividades; finalmente se inició la inclusión de las relaciones CTS-A en las clases de ciencias y como ya se mencionó, se dio mayor importancia a los contextos sociales y culturales de los estudiantes. 


\section{Análisis permanente (b): Equipos colaborativos de trabajo Análisis cualitativo mediante el uso del Software ATLAS.ti.}

La estrecha relación existente entre las actitudes y los saberes (planos cognitivo y cognoscitivo) es la que finalmente determina las acciones y hechos durante la práctica docente. El docente actúa a partir de sus preconceptos, concepciones, objetivos personales, creencias, entre otros muchos factores, y finalmente la suma de todas estas variables dan características particulares a la acción docente.

Generar espacios para el tratamiento de situaciones problema sobre la enseñanza de la química en equipos colaborativos de profesores noveles y expertos, es la parte trascendental que produce los cambios actitudinales, conceptuales y procedimentales en el grupo de docentes con el que se trabajó. En otras palabras, estos cambios son los que en suma, podemos considerar como cambios didácticos que representan las variaciones en el CDC de los profesores. Es por ello que la variable interviniente ha sido un programa de actividades (elaborado por los autores de esta investigación) en el que se abordaron las situaciones problemas en la enseñanza de la química.

Para el desarrollo de las actividades dentro de los equipos colaborativos de trabajo, se establecieron unos parámetros de seguimiento.

1. Identificar los problemas cotidianos existentes en las Clases de Química.

2. Verbalizar estos problemas dentro de los equipos colaborativos de trabajo.

3. Exposiciones, presentaciones y talleres dirigidos por parte del grupo de investigación para abordar los problemas tratados.

4. Complementación por parte de todo el equipo colaborativo de trabajo, por medio de la realización de lecturas de documentos seleccionados.

5. Sugerir a los profesores noveles y expertos que incluyan los nuevos conocimientos a sus prácticas. La implementación o no de estas sugerencias, es analizada a través de grabaciones de las actividades académicas - explicacio- nes, talleres, evaluaciones, laboratorios, etc. Finalmente, aplicada una entrevista de cierre y sus resultados complementados en la aplicación de un cuestionario escala Liker, permitió dar cuenta de los cambios didácticos logrados, los cuales en suma, en el contexto de esta investigación, nos dan luces para identificar el CDC de cada uno de los agentes participantes en este trabajo. Por efectos de espacio, únicamente se presentan a continuación los análisis logrados para uno de los docentes nóveles participantes.

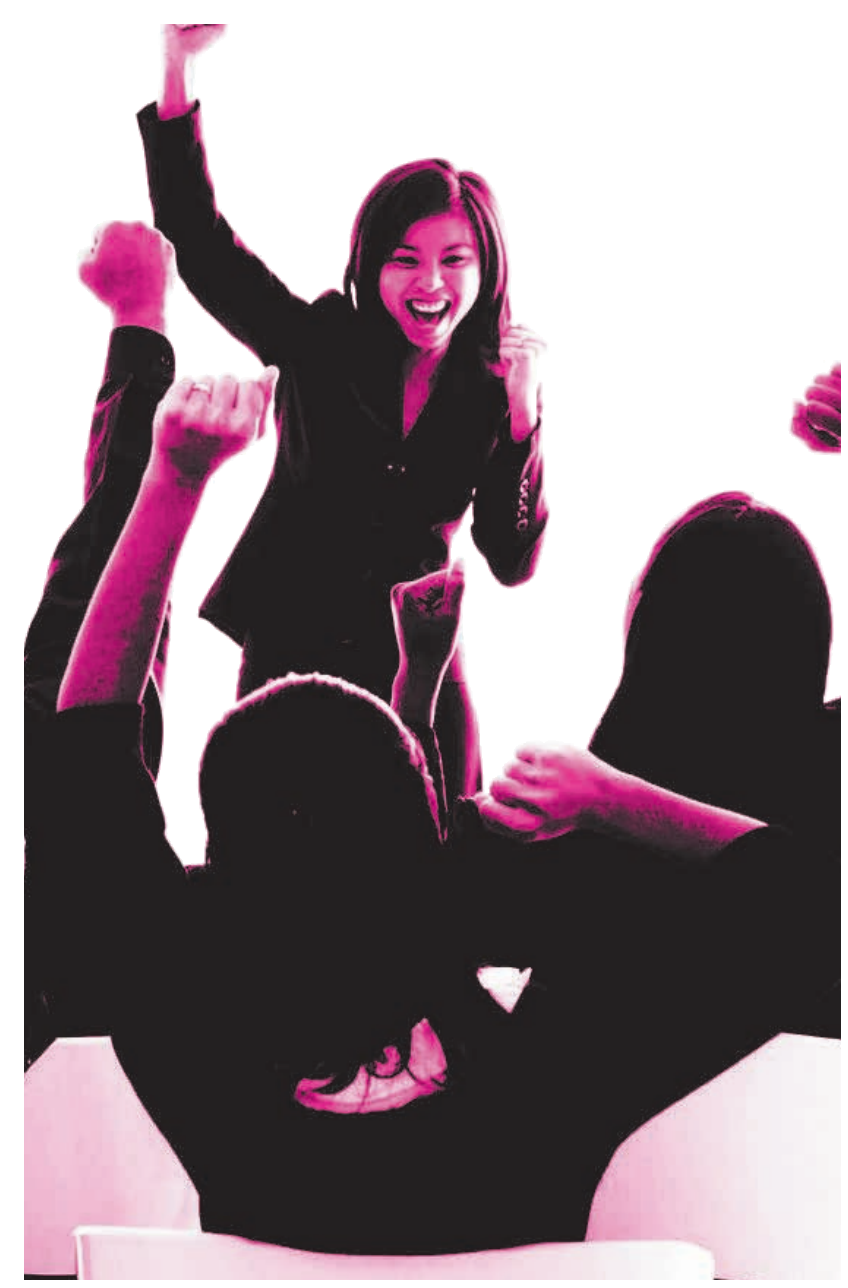




\section{Bibliografía}

Abell, S. (2007). "Research on science teachers' knowledge". En: Handbook of research on science education. (Abell, S. K.; Lederman, N. G.; Mahwa, editores), New Jersey: Lawrence Erlbaum Associates.

Aduriz, A.; Izquierdo, M.; Estany, A. (2002). "Una propuesta para estructurar la enseñanza de la filosofía de la ciencia para el profesorado de la ciencia en formación”. En: Revista Enseñanza de las Ciencias, volumen 20 (3); pp. $465-476$.

Ariza, L.G.; Parga, D. (2008). “El Conocimiento Didáctico del Contenido de los profesores de química en Relación con la Combustión". Primer Coloquio Internacional y Tercer Nacional de Didáctica de las Ciencias. Memorias, Ibagué: Universidad del Tolima.

Baxter, J. A. y Lederman, N. G. (1999). "Assessment and measurement of pedagogical content knowledge". En Examining pedagogical content knowledge: the construct and its implications for science education. (Gess-Newsome, J.; Lederman, N. G., editores), Dordrecht, The Netherlands: Kluwer Academic Publishers.

Bolívar, A. (2005). "Conocimiento didáctico del contenido y didácticas específicas". En: Revista de Currículum y Formación del Profesorado, pp. 1-39.

Carter, K. (1990). “Teachers' Knowledge and Learning to Teach”. En: Handbook of Reasearch on Teacher Education. (Houston, R. editor). Macmillam, pp. 291-310, New York.

Clermont, C.P., Krajcik, J.S., \& Borko, H. (1993). "The influence of an intensive in-service workshop on pedagogical content knowledge growth among novice chemical demonstrators". En: Journal of Research in Science Teaching, volumen 30 , pp. 21-43.

Clermont, C. P., Borko, H., \& Krajcik, J. S. (1994). "Comparative study of the pedagogical content knowledge of experienced and novice chemical demonstrators". En: J ournal of Research in Science Teaching, volumen 31, pp. 419-441.

Dawkins, K., Butler, S. (2001). Analyzing preservice chemistry teacher's pedagogical content knowledge regarding mole concept, Proceedings of the annual meeting of the Association for Teacher Education in Europe. Stockholm. Disponible en: http://www.soe.ecu.edu/csmte/research.htm, documento revisado en agosto 30 de 2011.

De Jong, O.; Driel, J. H. Van, \& Verloop, N. (2005). "Pre-service teachers' pegagogical content knowledge of using particle models in teaching chemistry". En: J ournal of Research in Science Teaching, pp. 947-964.

Elbaz, F. (1983). Teacher Thinking. A Study of Practical Knowledge. London: Croom Helm.

Garritz, A. (2006). "Historia y retos de la formación de profesores (Algo más sobre Lee Shulman)". En: Educación Química, volumen $17(3)$, pp. 322-326.

Gil, D. (1991). "¿Qué hemos de saber y saber hacer los profesores de ciencias? Intento de síntesis de las aportaciones de la investigación didáctica”. En: Revista Enseñanza de las Ciencias. volumen 9(1), pp. 69 - 77.

Grossman, P., Wilson, S. and Shulman, L. (1989). "Teachers of Substance: Subject Matter Knowledge for Teaching". En: Knowledge Base for the Beginning Teacher (Reynolds, M., editor), pp. 23-36, New York: Pergamon Press. 
Leinhardt, G. And smith, D. (1985). "Expertise in mathematics instruction: Subject matter Knowledge”. En: J ournal of Educational Psychology, volumen 77 (3), pp. 247-271.

Loughran, J. J.; Berry, A.; Mulhall, P. (2006). Understanding and developing science teachers' pedagogical content knowledge. Rotterdam, The Netherlands: Sense Publishers.

Marcelo, C. (1992). Como conocen los profesores la materia que enseñan. Algunas contribuciones de la investigación sobre Conocimiento Didáctico del Contenido, Ponencia presentada en el Congreso Internacional Las didácticas específicas en la formación del profesorado. 6 de julio, Santiago de Compostela.

Marcelo, C. (1987). El Pensamiento del Profesor. Barcelona: CEAC.

Mellado, V. (1994). Análisis del conocimiento didáctico del contenido, en profesores de ciencias de primaria y secundaria en formación inicial. Facultad de ciencias de la Educación, Universidad de Sevilla, versión digital: http://fondosdigitales.us.es/tesis/tesis/620/analisis-del-conocimiento-didactico-del-contenido-en-profesores-de-ciencias-de-primariay-secundaria-en-formacion-inicial/

Mora, W. M.; Parga, D.L. (2007). "El conocimiento didáctico del contenido en química: integración de las Tramas de contenido / histórico - epistemológicas con las Tramas de Contexto / Aprendizaje”. En: Tecné, Episteme y Didaxis. TE $\Delta$, número 24, pp. 54-74.

Mosquera, C. J. (2008). La didáctica de las ciencias. Fundamento del conocimiento profesional de los profesores de ciencia. En: Revista Electrónica el Educador. pp. 16 -18.

Mosquera, C. J. (2009). "Propuesta de proyecto de Investigación”, presentada al Centro de Investigaciones y Desarrollo Científico, Universidad Distrital "Francisco José de Caldas", Convocatoria 001 - 2009.

Peterson, P. (1988). “Teachers' and Students' Cognitional Knowledge for Classroom Teaching and Learning”. En: Educational Researcher, volumen 17 (5), pp. 5-14.

Shulman, L. S. (1986). "Those who understand: knowledge growth in teaching”. En: Educational Researcher, 15(2), 4-14. Traducción castellana (2005): “El saber y entender de la profesión docente”. En: Estudios Públicos, volumen 99, pp. 195-224.

Shulman, L. S. (1987). "Knowledge and teaching: foundations of the new reform". Harvard Educational Review, 57(1), 1-22. Traducción castellana (2005): "Conocimiento y enseñanza: fundamento de la nueva reforma". En: Revista de Currículum y Formación de Profesorado, volumen 9(2), versión digital: http://www.ugr.es/ recfpro/rev92ART1.pdf.

Stake, R. E. (1998): Investigación con estudio de casos. Madrid: Editorial Morata.

Talanquer, V.; D., A. Stronger. (2003). "Role for Science Departments in the Preparation of Future Chemistry Teachers". En: J ournal Chemistry Education, volumen 80(10), pp. 1168- 1171.

Treagust, D. F. y Mamiala, T. L. (2003). "The role of submicroscopic and symbolic representations in chemical explanations”. En: International J ournal of Science Education, volumen 25(11). pp. 1353-1368.

Van Driel, J. H.; Verloop, N.; De Vos, W. (1998). “Developing science teachers' pedagogical content knowledge”. En: J ournal of Research in Science Teaching, volumen 35(6), pp. 673-695. 
Van Driel, J. H.; De Jong, O. (2001). "Investigating the development of pre-service teachers' pedagogical content knowledge", Annual Meeting of the National Association for Research in Science Teaching. St. Louis: MO.

Veal, W. R. (1998). "The Evolution of Pedagogical Content Knowledge in Prospective Secondary Chemistry Teachers", Annual Meeting of the National Association of Research in Science Teaching. San Diego, CA, pp. 1-47, disponible en: http://www.educ.sfu.ca/narstsite/conference/98conference/veal2.pdf. Documento revisado el 30 de agosto de 2011.

Veal, W. R.; Makinster, J. G. (1999). "Pedagogical Content Knowledge Taxonomies" En: Electronic J ournal of Science Education, volumen 3(4), disponible en: http://unr.edu/homepage/crowther/ejse/vealmak.html. Documento revisado el 30 de agosto de 2011.

Wilson, S.; Shulman, L. (1987). "150 Different Ways of Knowing: Representations of Knowledge in Teaching", En: Exploring Teacher Thinking. (En Calderhead, J.) Eastbourne, England, pp. 104-124. 